\title{
A novel mechanism for hypofibrinolysis in diabetes: the role of complement $\mathbf{C 3}$
}

\author{
K. Hess • S. H. Alzahrani • M. Mathai • V. Schroeder - A. M. Carter • G. Howell • \\ T. Koko • M. W. J. Strachan • J. F. Price • K. A. Smith • P. J. Grant • R. A. Ajjan
}

Received: 20 May 2011 / Accepted: 12 August 2011 / Published online: 15 September 2011

(C) Springer-Verlag 2011

\begin{abstract}
Aims/hypothesis Impaired fibrin clot lysis is a key abnormality in diabetes and complement $\mathrm{C} 3$ is one protein identified in blood clots. This work investigates the mechanistic pathways linking $\mathrm{C} 3$ and hypofibrinolysis in diabetes using ex vivo/in vitro studies.

Methods Fibrinolysis and C3 plasma levels were determined in type 1 diabetic patients and healthy controls, and the effects of glycaemia investigated. C3 incorporation into fibrin clots and modulation of fibrinolysis were analysed by
\end{abstract}

K. Hess $\cdot$ S. H. Alzahrani $\cdot$ V. Schroeder $\cdot$ A. M. Carter $\cdot$ T. Koko $\cdot$

K. A. Smith · P. J. Grant • R. A. Ajjan $(\triangle)$

Division of Cardiovascular and Diabetes Research,

Multidisciplinary Cardiovascular Research Centre, Leeds Institute

for Genetics, Health and Therapeutics, LIGHT Laboratories,

Clarendon Way, University of Leeds,

Leeds LS2 9JT, UK

e-mail: r.ajjan@leeds.ac.uk

K. Hess

Department of Internal Medicine I,

University Hospital RWTH Aachen,

Aachen, Germany

\section{Mathai}

Department of Paediatrics, Bradford Royal Infirmary,

Bradford, UK

G. Howell

Faculty of Biological Sciences, University of Leeds,

Leeds, UK

M. W. J. Strachan

Metabolic Unit, Western General Hospital,

Edinburgh, UK

J. F. Price

Centre for Population Health Sciences, University of Edinburgh,

Edinburgh, UK
ELISA, immunoblotting, turbidimetric assays and electron and confocal microscopy.

Results Clot lysis time was longer in diabetic children than in controls ( $599 \pm 18$ and $516 \pm 12$ s respectively; $p<0.01$ ), C3 levels were higher in diabetic children $(0.55 \pm 0.02$ and $0.43 \pm$ $0.02 \mathrm{~g} / 1$ respectively; $p<0.01$ ) and both were affected by improving glycaemia. An interaction between $\mathrm{C} 3$ and fibrin was confirmed by the presence of lower protein levels in sera compared with corresponding plasma and $\mathrm{C} 3$ detection in plasma clots by immunoblot. In a purified system, C3 was associated with thinner fibrin fibres and more prolongation of lysis time of clots made from fibrinogen from diabetic participants compared with controls $(244 \pm 64$ and $92 \pm 23 \mathrm{~s}$ respectively; $p<0.05$ ). Confocal microscopy showed higher C3 incorporation into diabetic clots compared with controls, and fully formed clot lysis was prolonged by $764 \pm 76$ and $428 \pm 105 \mathrm{~s}$ respectively $(p<0.05)$. Differences in lysis, comparing diabetes and controls, were not related to altered plasmin generation or C3-fibrinogen binding assessed by plasmon resonance.

Conclusions/interpretation $\mathrm{C} 3$ incorporation into clots from diabetic fibrinogen is enhanced and adversely affects fibrinolysis. This may be one novel mechanism for compromised clot lysis in diabetes, potentially offering a new therapeutic target.

Keywords Clot structure - Complement C3 · Fibrinolysis . Type 1 diabetes

$\begin{array}{ll}\text { Abbreviations } \\ \text { CRP } & \text { C-reactive protein } \\ \text { FXIII } & \text { Factor XIII } \\ \text { PAI-1 } & \text { Plasminogen activator inhibitor 1 } \\ \text { SPR } & \text { Surface plasmon resonance } \\ \text { tPA } & \text { Tissue plasminogen activator }\end{array}$




\section{Introduction}

The formation of fibrin clots represents the final step in the atherothrombotic process and individuals with diabetes are at increased risk of arterial occlusive disease [1]. Fibrin network structure plays a crucial role in determining predisposition to atherothrombotic events as clots with thin fibres and small pores are associated with premature and more severe cardiovascular disease [2-4]. This is due to more difficult lysis of clots composed of such compact structure [5], which in turn is related to differential incorporation of various plasma proteins [6]. Previous work has shown more compact fibrin clots in type 1 diabetic patients compared with healthy controls [7], and limited evidence suggests a thrombotic and hypofibrinolytic state in individuals with type 1 diabetes by mechanisms that are not entirely understood [8]. Manipulating glycaemia in type 1 diabetes is associated with fewer thrombotic fibrin clots but the effect on fibrinolysis has not been studied $[9,10]$.

Chronic low grade inflammation is associated with atherothrombosis and C-reactive protein (CRP), as well as complement $\mathrm{C} 3$ plasma levels, are predictive of arterial thrombotic events $[11,12]$. In addition to the inflammatory role of complement, different complement proteins interact directly with the coagulation and thrombotic pathways. Recent work has shown molecular communications between the complement system and thrombotic proteins but only the immunemodulating properties of coagulation proteins were studied [13]. Limited studies suggest interactions in the opposite direction as terminal complement complexes enhance tissue factor expression [14] and complement proteins have also been implicated in modulating fibrin gel characteristics [15].

Using a proteomics approach, the presence of $\mathrm{C} 3$ in the fibrin clot has been demonstrated, which may modulate neutrophil recruitment into the site of injury and prolong clot lysis [16]. Furthermore, C3 plasma levels correlate with clot lysis time after correcting for fibrinogen and plasminogen activator inhibitor 1 (PAI-1) levels [17], which may be one mechanism for the association between $\mathrm{C} 3$ and cardiovascular events [12, 18-20].

Given hypofibrinolysis in diabetes, the interaction between coagulation and complement proteins, and the correlation between $\mathrm{C} 3$ plasma levels and fibrin clot lysis in non-diabetic individuals, we hypothesise that $\mathrm{C} 3$ has a direct role in modulating fibrin network lysis in individuals with diabetes. Therefore, the aim of the present study was to investigate (1) plasma clot lysis and potential interactions with $\mathrm{C} 3$ levels in younger individuals with type 1 diabetes before the development of complications; (2) the effects of improving glycaemic control on lysis efficiency and $\mathrm{C} 3$ plasma levels; (3) mechanisms for the compromised clot lysis in diabetes and the direct role of $\mathrm{C} 3$ using purified systems. To minimise the effects of confounding variables, and address the role of glycaemia, children and young adults with type 1 diabetes were studied.

Understanding the mechanisms for impaired fibrinolysis in diabetes will provide novel insights into pathways responsible for increased thrombosis risk in this population, potentially offering alternative treatment targets.

\section{Research design and methods}

Subjects and blood sampling

Thirty children with type 1 diabetes and 17 matched controls were recruited as a case-control cohort. Eighteen adult type 1 diabetic individuals with poor diabetes control $\left(\mathrm{HbA}_{1 \mathrm{c}}>8.5 \%\right.$ [ $>69 \mathrm{mmol} / \mathrm{mol}])$ were recruited in a longitudinal interventional study. Exclusion criteria included individuals with known cardiovascular disease, hypertension, nephropathy, autoimmune disorders, significant retinopathy or any treatment other than insulin. The study was approved by the local research ethical committee and appropriate consent was obtained from all participants. Patients were instructed to have a light breakfast (to avoid hypoglycaemia before their appointment), generally consisting of two slices of toast and injection of appropriate quantity of short-acting insulin, and blood samples were collected mid-morning. Samples were collected without a tourniquet into citrated tubes, plasma separated and stored in aliquots at $-40^{\circ} \mathrm{C}$.

In the young adult group, individuals were asked to record capillary blood glucose regularly and glycaemic control was optimised by adjusting insulin doses, through regular clinic appointments and 2-4 weekly contact by phone or email as per patient preference. The aim was to improve diabetes control, stabilise this improvement and collect repeat blood samples at 6-8 months to analyse the effects of medium-term, rather than short-term diabetes control.

\section{Analysis of plasma samples}

Plasma protein levels Plasma complement C3 was determined using an ELISA Kit (GenWay Biotech, San Diego, CA, USA) and high sensitivity native CRP was analysed as previously described [20]. PAI-1 was assessed by ELISA (Invitrogen, Paisley, UK). Fibrinogen levels were determined by the Clauss method [21].

Fibrinolysis of plasma samples A turbidity assay was conducted to assess fibrinolysis as previously described [22]. Lysis time was measured from full clot formation to $50 \%$ clot lysis.

C3 incorporation into plasma clots We investigated C3 levels in plasma and corresponding sera as follows: either 
$25 \mu$ activation mix with a final concentration of $5 \mathrm{U} / \mathrm{ml}$ human thrombin (Calbiochem, Nottingham, UK) and $15 \mathrm{mmol} / 1 \mathrm{CaCl}_{2}$ or the same volume of sample buffer (100 mmol/l NaCl, $50 \mathrm{mmol} / \mathrm{l}$ Tris, $\mathrm{pH} \mathrm{7.4)}$ was added to $125 \mu$ individual plasma samples of randomly chosen patients with diabetes $(n=10)$ and healthy controls $(n=10)$. Samples were incubated for $4 \mathrm{~h}$ at $37^{\circ} \mathrm{C}$ after which the resulting thrombus was removed manually, and C3 levels measured by ELISA in recovered sera and corresponding plasma.

To confirm C3 incorporation into the fibrin clot, $5 \mu \mathrm{l}$ of activation mix containing $5 \mathrm{U} / \mathrm{ml}$ human thrombin and $15 \mathrm{mmol} / 1 \mathrm{CaCl}_{2}$ was added to $45 \mu \mathrm{l}$ randomly chosen plasma samples. Samples were incubated for $4 \mathrm{~h}$ at $37^{\circ} \mathrm{C}$, after which the resulting clots were manually removed and extensively washed three times in sample buffer, followed by incubation at $95^{\circ} \mathrm{C}$ for $20 \mathrm{~min}$ in the presence of $16.7 \mu \mathrm{l}$ sample buffer and $6.7 \mu \mathrm{l}$ reducing buffer (Invitrogen). Samples were loaded onto 4-12\% SDS-PAGE gel (Invitrogen), and immunoblotted using a polyclonal rabbit antiC3 and HRP-labelled goat anti-rabbit as a secondary antibody (both Dako Cytomatic, Cambridge, UK). Purified C3 was used as a positive control. Similar experiments were conducted using polyclonal rabbit anti-CRP antibody and HRP-labelled goat anti-rabbit as a secondary antibody (both Dako Cytomatic).

\section{Analysis of plasma-purified proteins}

Protein purification Human C3 was purified from pooled healthy control plasma using a modified protocol from Van den Berg [23]. Briefly, plasma proteins were precipitated by polyethylene glycol 4000, followed by an anion exchange chromatography using Mono Q 5/50 GL (GE Healthcare, Bucks, UK) and gel filtration using Superose 12 10/300 GL (GE Healthcare).

Fibrinogen was purified by affinity chromatography using calcium-dependent IF-1 monoclonal antibody as previously described [6].

Plasminogen was purified from plasma samples by lysine sepharose affinity chromatography as previously described [24].

Integrity and purity of purified fibrinogen, C3 and plasminogen were assessed by SDS-PAGE and immunoblotting. C5 contamination of C3 preparations was ruled out by immunoblotting using C5-specific antibodies. All purified proteins were quantified by ELISA.

\section{Clot structure and lysis}

Turbidity assay These were performed as described elsewhere [6]. Briefly, pooled purified fibrinogen from diabetic and control participants ( $n=9$ each) was used at $2.94 \mu \mathrm{mol} / 1$, $\mathrm{C} 3$ at $0.5 \mathrm{~g} / 1$ (or BSA at $2 \mathrm{mg} / \mathrm{ml}$ ), in the presence of $10 \mu \mathrm{g} / \mathrm{ml}$ Factor XIII (FXIII) and $6 \mu \mathrm{g} / \mathrm{ml}$ plasminogen.

Scanning electron microscopy Clots were prepared using purified fibrinogen as previously described, in the presence and absence of C3 [6]. Fibrinogen was used at $1.44 \mu \mathrm{mol} / 1$, $\mathrm{C} 3$ at $0.245 \mathrm{~g} / 1$ and samples clotted in the presence of 0.5 $\mathrm{U} / \mathrm{ml}$ thrombin, $2.5 \mathrm{mmol} / 1 \mathrm{CaCl}_{2}$ and $4.9 \mu \mathrm{g} / \mathrm{ml} \mathrm{FXIII}$. Fibre diameters of all clots were measured with image analysis software package ImageJ 1,23y (National Institutes of Health, Bethesda, MD, USA). In all, 100 fibrin fibres per sample were measured.

Laser scanning confocal microscopy Purified C3 was fluorescent-labelled using the DyLightTM Labelling Kits (Pierce, Biotechnology, Thermo Scientific, Loughborough, UK). Diabetes or control fibrinogen (1.44 $\mu \mathrm{mol} / \mathrm{l})$ was added to an activation mix containing $0.05 \mathrm{U} / \mathrm{ml}$ human thrombin (Calbiochem), $4.9 \mu \mathrm{g} / \mathrm{ml} \mathrm{FXIII,} 5 \mathrm{mmol} / \mathrm{C} \mathrm{CaCl}_{2}$, $0.105 \mu \mathrm{mol} / 1$ fluorescent labelled fibrinogen (Alexa Fluor Dye; Invitrogen) and $0.245 \mathrm{~g} / 1$ inhouse labelled C3, with $15 \mu \mathrm{l}$ of the mixture loaded onto an Ibidi slide (Applied Biophysics, New York, NY, USA). Each experiment was repeated on at least four different occasions (each in duplicate) with identical confocal settings applied to diabetes and control clots. Fibrin networks were visualised by a $\times 40 / 1.3$ oil ph3 objective LSM 510 META microscope from Carl Zeiss (Welwyn Garden City, UK), and Z-stacks were prepared of 11 images at $10 \mu \mathrm{m}$ intervals and/or at $2 \mu \mathrm{m}$ intervals (clot thickness 100 and $20 \mu \mathrm{m}$ respectively). To quantify C3 incorporation into the clot, distribution of pixel intensity within fibrin fibres was measured. Images were split into green (fibrinogen) and red (C3) channels, and the green image was thresholded to remove background signal. Areas of clot fibres were superimposed on the red channel and fluorescence intensity from each pixel was determined, followed by data display as histograms showing pixel intensity.

Real-time fibrinolysis was studied by adding $10 \mu \mathrm{l}$ mixture of $10 \mathrm{nmol} / 1$ tissue plasminogen activator (tpA) and $0.2 \mathrm{mmol} / \mathrm{l}$ plasminogen in Tris-buffered saline to the border of the fibrin clot and subsequently serial photographs were taken of the lysis front.

Fibrinogen-C3 interaction

Surface plasmon resonance (SPR) experiments employed a Biacore 3000 system as previously described [25]. Briefly, 500 RU of pooled purified control or diabetic fibrinogen $(n=9$ each) was immobilised on a CM5 sensor and serial dilutions of purified C3 (0.019-10 nmol/l) were injected over the 
control $(\mathrm{Fc} 2)$ and diabetic fibrinogen $(\mathrm{Fc} 4)$. When required, fibrinogen surface was converted to fibrin by injecting $5 \mathrm{U} / \mathrm{ml}$ thrombin, followed by washing with $1 \mathrm{~mol} / \mathrm{l} \mathrm{NaCl}$ to remove $\mathrm{FpA} / \mathrm{FpB}$. Kinetics analysis was performed using BIAevaluation software 4.1. Each data set was fitted to a $1: 1$ Langmuir interaction model to obtain the equilibrium dissociation constants $\left(K_{\mathrm{D}}\right)$ for this interaction $(n=3)$. For reverse orientation, $100 \mathrm{RU}$ of purified $\mathrm{C} 3$ was immobilised and serial dilution of $0.39-50 \mathrm{nmol} / 1$ control and diabetic fibrinogen was injected in ascending order. For this orientation, each data set was fitted to a bivalent interaction model.

Plasminogen activation assay

This was performed using protein purified from a pool of healthy control plasma according to a modified protocol by Bobbink et al. [26]. Briefly, microtitre plates were coated with $0.23 \mu \mathrm{mol} / 1$ fibrinogen either from controls or diabetic patients in the presence or absence of $0.04 \mu \mathrm{g} / \mathrm{l} \mathrm{C} 3$. Plates were blocked (50 mmol/l Tris-HCl, $140 \mathrm{mmol} / \mathrm{l} \mathrm{NaCl}, 3 \%$ BSA, $0.01 \%$ Tween 20 ), incubated with $1 \mathrm{U} / \mathrm{ml}$ thrombin and $75 \mathrm{nmol} / \mathrm{l}$ plasminogen, $12 \mathrm{nmol} / \mathrm{l} \mathrm{tPA}$ and $0.8 \mathrm{mmol} / \mathrm{l}$ plasmin specific chromogenic substrate S2251 (Chromogenic, Epsom, UK) was added. S2251 hydrolysis was monitored via kinetic absorbance readings at $405 \mathrm{~nm}$ on an ELX-808 IU ultramicroplate reader (Bio Tek Instruments, Winooski, VT, USA).

\section{Statistical analysis}

Results are presented as mean $\pm \mathrm{SEM}$. Between-group comparisons of normally distributed variables were carried out by independent sample Student's $t$ tests, whereas comparisons of non-normally distributed variables were carried out by Mann-Whitney $U$ tests. Pearson and Spearman coefficients were applied to assess correlations between normally and non-normally distributed variables respectively.

\section{Results}

Subject details

A case-control study was performed on children with type 1 diabetes and age-matched controls, whereas a longitudinal study was conducted on young adults with type 1 diabetes (details provided in Tables 1 and 2 respectively). Diabetes treatment was intensified in the latter group and repeat blood samples taken after a mean of $7.3 \pm 0.9$ months. The majority of patients were on basal bolus regime with short-acting insulin dose varied according to food carbohydrate content. The method of insulin delivery was kept constant and diabetes control was optimised through regular contact by phone or email, to encourage compliance with insulin injections and guide on dose adjustment.

C3 and CRP plasma levels in patients with type 1 diabetes

C3 plasma levels were higher in children with diabetes compared with age- and weight-matched healthy controls $(0.55 \pm 0.02$ and $0.43 \pm 0.02 \mathrm{~g} / 1$ respectively; $p<0.001)$, with similar findings observed for CRP levels $(0.72 \pm 0.20$ and $0.50 \pm 0.29 \mathrm{mg} / \mathrm{l}$ respectively; $<0.05$; Fig. $1 \mathrm{a}$ and b). In young adults, reduction in $\mathrm{HbA}_{1 \mathrm{c}}$ from $10.5 \%$ to $9.2 \%$ (91-77 mmol/ mol) was associated with a decrease in $\mathrm{C} 3$ levels from $0.65 \pm$ 0.04 to $0.54 \pm 0.02 \mathrm{~g} / 1(p<0.05)$. CRP levels also decreased
Table 1 Clinical variables of control and type 1 diabetic individuals

NA, not applicable

\begin{tabular}{lllr}
\hline Variable & $\begin{array}{l}\text { Children with type } 1 \\
\text { diabetes }(n=30)\end{array}$ & $\begin{array}{l}\text { Children without type } 1 \\
\text { diabetes }(n=17)\end{array}$ & $p$ value \\
\hline Age (years) & $14.5 \pm 0.3$ & $13.6 \pm 0.4$ & 0.09 \\
Sex $(\mathrm{M} / \mathrm{F})$ & $20 / 10$ & $8 / 9$ & \\
Time since diagnosis (years) & $6.32 \pm 0.5$ & $53.4 \pm 3.6$ & 0.32 \\
Weight $(\mathrm{kg})$ & $57.9 \pm 1.9$ & & \\
HbA $_{1 \mathrm{c}}(\%$ mmol/mol] $)$ & $9.6 \pm 0.4[81 \pm 5]$ & 0 & \\
Current smoker & 0 & $\mathrm{NA}$ & \\
Insulin treatment & 1 & $\mathrm{NA}$ & \\
Twice daily mixed insulin & 21 & $\mathrm{NA}$ & 0.27 \\
Basal bolus regime & 8 & $6.94 \pm 0.32$ & \\
Insulin pump & $8.67 \pm 0.38$ & $791 \pm 124$ & \\
Plasma fibrinogen $(\mu \mathrm{mol} / \mathrm{l})$ & $643 \pm 92$ & 0 & \\
PAI-1 (pg/ml) & 0 & & \\
Microvascular disease & & & \\
\hline
\end{tabular}


Table 2 Clinical variables of young adults with type 1 diabetes mellitus

$\mathrm{BR}$, background retinopathy

\begin{tabular}{llll}
\hline Variable & Pre intervention $(n=18)$ & Post intervention $(n=18)$ & $p$ value \\
\hline Age (years) & $23 \pm 5$ & & \\
Sex $(\mathrm{M} / \mathrm{F})$ & $7 / 11$ & & \\
Time since diagnosis (years) & $12.4 \pm 1.5$ & $72.1 \pm 3.6$ & 0.08 \\
Weight $(\mathrm{kg})$ & $71.0 \pm 3.3$ & $9.2 \pm 0.4[77 \pm 5]$ & 0.02 \\
HbA $_{1 \mathrm{c}}(\%$ [mmol/mol]) & $10.5 \pm 0.5[91 \pm 5]$ & 5 & \\
Current smoker & 5 & & \\
Insulin treatment & & 1 & 0.35 \\
Twice daily mixed insulin & 1 & 16 & 0.67 \\
Basal bolus regime & 16 & 1 & \\
Insulin pump & 1 & $7.26 \pm 0.70$ & \\
Plasma fibrinogen $(\mu \mathrm{mol} / \mathrm{l})$ & $7.58 \pm 0.67$ & $403 \pm 72$ & \\
PAI-1 (pg/ml) & $453 \pm 103$ & 0 & $4(\mathrm{BR})$ \\
Macrovascular disease & 0 & & \\
Microvascular disease & $4(\mathrm{BR})$ & & \\
\hline
\end{tabular}

from $2.0 \pm 0.55$ to $1.3 \pm 0.24 \mathrm{mg} / \mathrm{l}$, but this was not significant ( $p=0.69$; Fig. 1c and d). Similarly, fibrinogen and PAI-1 levels did not significantly alter after improving glycaemic control (fibrinogen: $7.58 \pm 0.67$ and $7.26 \pm 0.70 \mu \mathrm{mol} / 1$; PAI-1: $453 \pm$ 103 and $403 \pm 72 \mathrm{pg} / \mathrm{ml}$ respectively, $p>0.1$ for both).

a

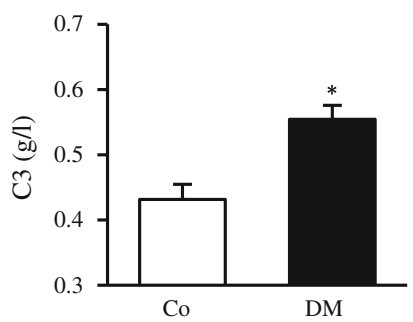

b

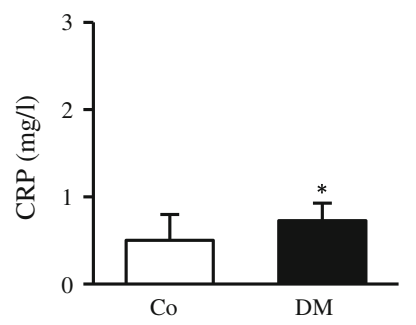

C3, but not CRP, levels correlated with variables of clot lysis in patients with type 1 diabetes mellitus

Lysis time in diabetic children was significantly longer compared with controls $(599 \pm 18$ and $516 \pm 12$ s respec-

C

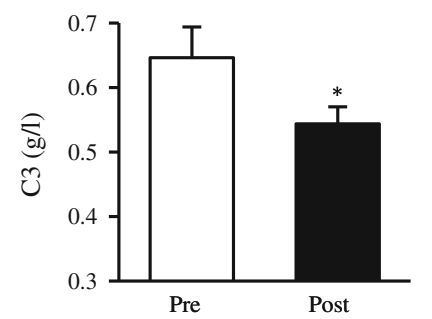

d

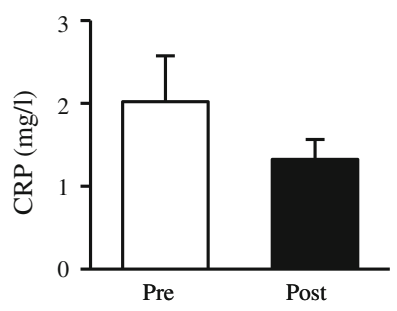

e

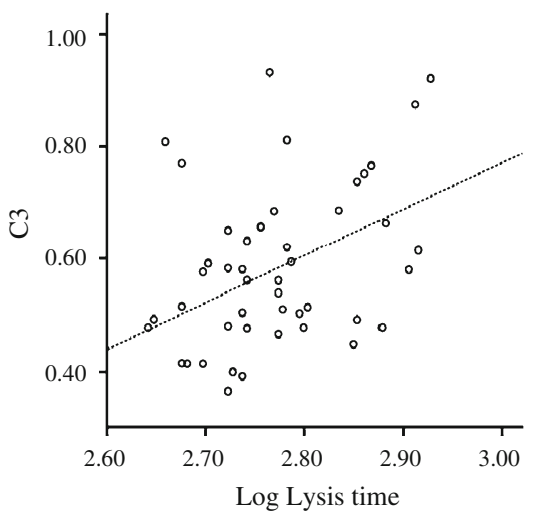

$\mathbf{f}$

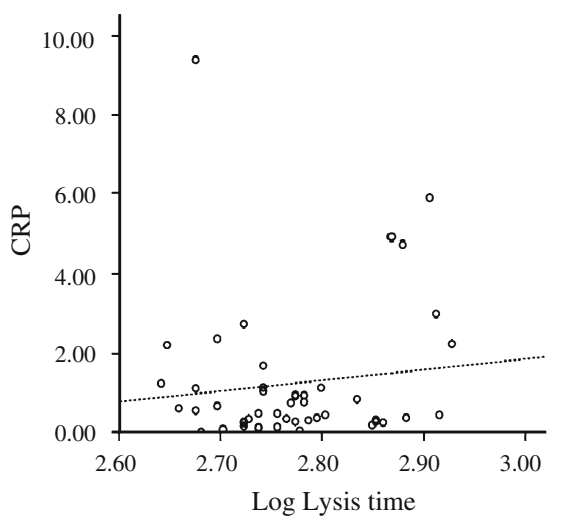

g

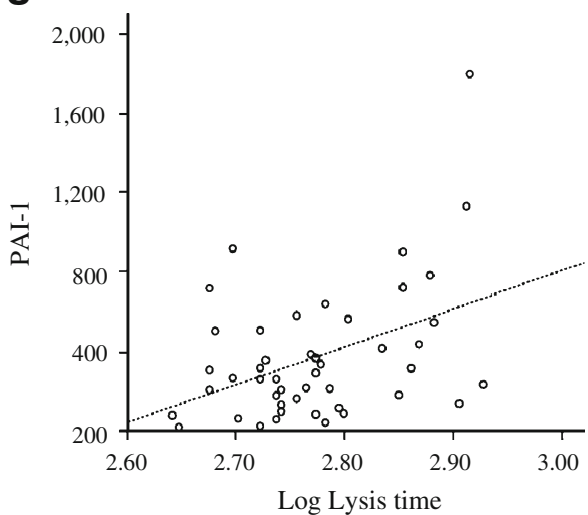

Fig. 1 Complement C3 and CRP levels. a, b C3 and CRP plasma levels in 30 children with type 1 diabetes (DM) and 17 healthy controls (Co). c, $\mathbf{d ~ C} 3$ and CRP plasma levels in 18 adults with type 1 diabetes before (pre, $\mathrm{HbA}_{1 \mathrm{c}} 10.5 \pm 0.5 \%[91 \pm 4.9 \mathrm{mmol} / \mathrm{mol}]$ ) and after improving glycaemic control (post, $\mathrm{HbA}_{1 \mathrm{c}} 9.2 \pm 0.4 \% \quad[77 \pm 4.7 \mathrm{mmol} / \mathrm{mol}]$ ). Median and SEM presented. ${ }^{*} p<0.05$. e-g Correlation between clot lysis time and C3, CRP and PAI-1 $(r=0.38 ; p=0.01 ; r=0.12 ; p=0.49$ and $r=0.42 ; p=0.004$ respectively) 
tively; $p<0.01)$. Improving glycaemic control in young adults shortened lysis time from $673 \pm 34$ to $600 \pm 23 \mathrm{~s}$ ( $p<$ $0.05)$.

C3 plasma levels correlated with lysis time in patients with diabetes $(r=0.38 ; p<0.01$; Fig. 1e), whereas CRP levels failed to show such a correlation $(r=0.12, p=0.42$; Fig. 1f). Compared with C3, PAI-1, the classical fibrinolytic inhibitor, showed similar correlation with lysis time at $r=$ $0.42(p<0.01)$. Moreover, the correlation between the change in $\mathrm{HbA}_{1 \mathrm{c}}$ and change of $\mathrm{C} 3$ before and after improving glucose levels in 18 individuals exhibited a trend $(r=0.45, p=0.06)$, suggesting an interaction between glycaemia and $\mathrm{C} 3$ plasma levels.
$\mathrm{C} 3$ is incorporated into the fibrin clot

C3 levels were significantly lower in sera compared with corresponding plasma of diabetic patients and controls $(28.2 \pm 5.5 \%$ and $20.3 \pm 5.1 \%$ reduction in $\mathrm{C} 3$ respectively; $p<0.01$ for each), suggesting incorporation of $\mathrm{C} 3$ into the clot (Fig. 2a). Although the percentage difference in $\mathrm{C} 3$ levels comparing plasma and serum samples was similar in diabetic and control individuals, the total difference in protein levels was $0.24 \pm 0.05$ and $0.12 \pm 0.03 \mathrm{~g} / 1$ respectively (Fig. 2b, $p=0.05$ ), indicating increased overall incorporation of $\mathrm{C} 3$ into diabetes clots. Immunoblotting of extensively washed plasma clots demonstrated $\mathrm{C} 3$ in both control

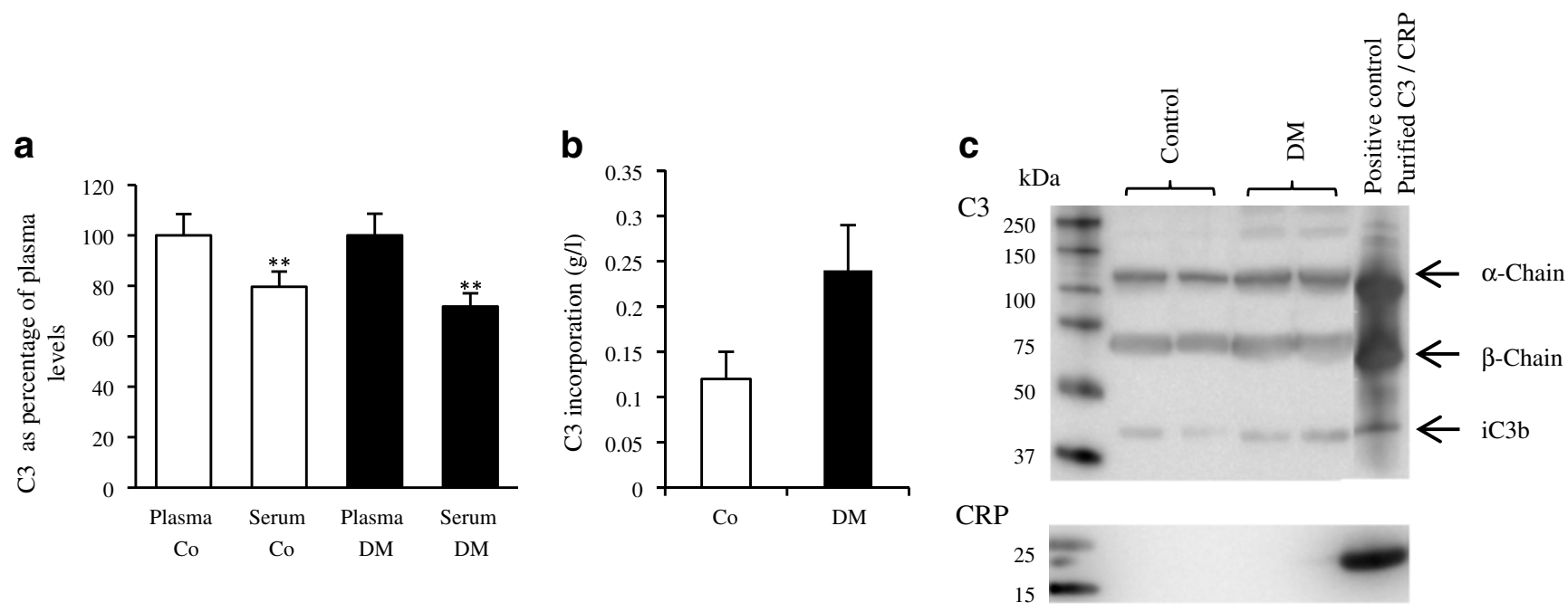

d

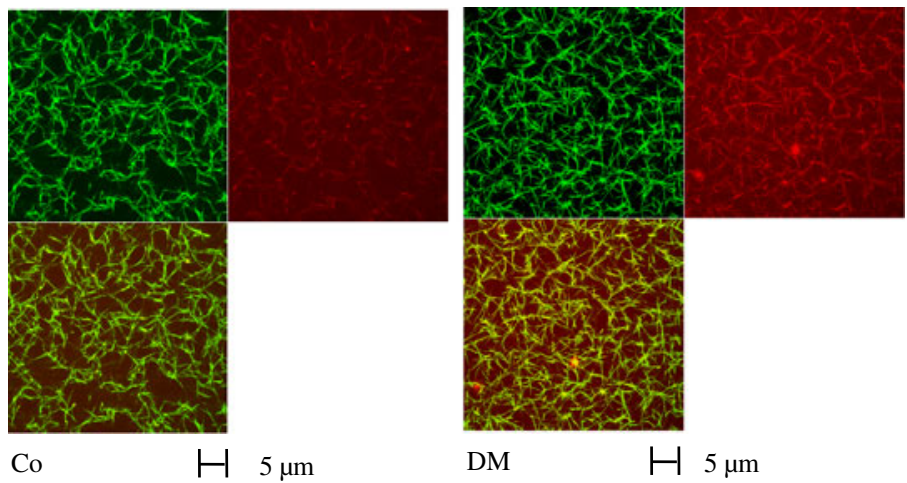

e

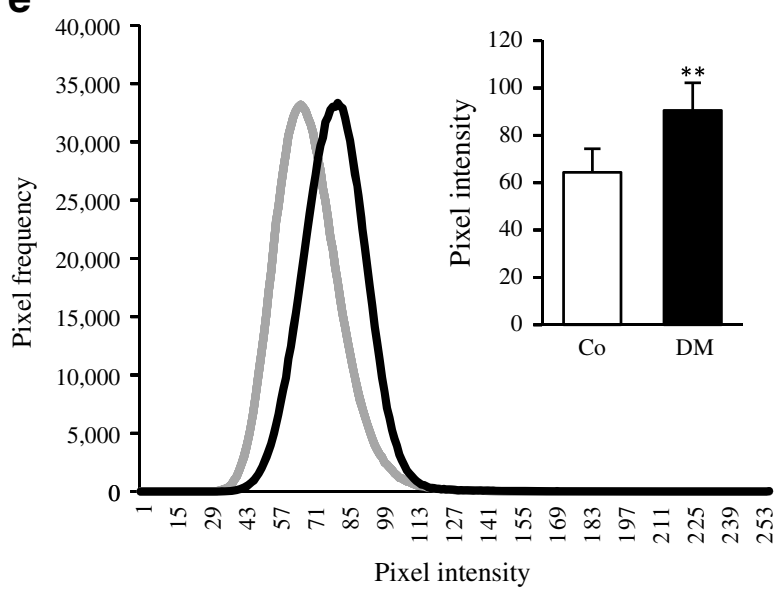

Fig. 2 Complement C3 incorporation into the fibrin clot. a Fibrin clots were made from plasma samples after recalcification and thrombin treatment as detailed in the Methods section. C3 levels were subsequently measured in individual plasma and corresponding sera from diabetes/control $(\mathrm{DM} / \mathrm{Co})$ individuals $(n=10 \mathrm{each})$, and results are presented as percentage $\mathrm{C} 3$ incorporation into the clot. b Total incorporation of C3 into individual DM and Co clots ( $n=10$ each; $p=$ 0.05). c Plasma clots were prepared from randomly chosen plasma samples of diabetes and control individuals, followed by manual removal of the clot and extensive washing. Clots were subsequently dissolved, ran on SDS-PAGE and immunoblotted using anti-C3 and anti-CRP antibodies. d Confocal microscopy using dual staining techniques in a purified system. Clots were made using Alexa 488 labelled fibrinogen (green) and Alexa 549 labelled C3 (red) and Z stack of 11 sections were taken over a depth of $20 \mu \mathrm{m}$. The overlay appears yellow. e Histogram of $\mathrm{C} 3$ demonstrating increased $\mathrm{C} 3$ incorporation into diabetic fibrin networks. Grey line, Co; black line, DM. Insert: mean pixel intensity $(n=5) .{ }^{* *} p<0.01$ 
and diabetes clots, whereas we failed to detect CRP in the clot (Fig. 2c).

C3 is visualised in the fibrin network with increased incorporation into clots formed from patients with diabetes

Fluorescent-labelled fibrinogen (green) and complement C3 (red) were used to prepare fibrin clots in a purified system to study incorporation of $\mathrm{C} 3$ into the fibrin network (Fig. 2d). Pooled fibrinogen was used from diabetes and control fibrinogen ( $n=9$ each), whereas C3 was from pooled control samples. Intensity of incorporated C3 was higher in diabetes clots compared with control and this was further quantified by measuring pixel intensity of $\mathrm{C} 3$ within each fibrin-positive structure as described in the methods. Histograms showed $41 \%$ increase $(n=5$ independent experiments, $p<0.01$ ) in mean fluorescence intensity of $\mathrm{C} 3$ in clots made using purified fibrinogen from diabetic patients compared with controls (Fig. 2e), indicating an increase in C3 incorporation into diabetic clots. This is consistent with plasma experiments above, confirming higher C3 incorporation into diabetes clots compared with controls.

C3 prolongation of clot lysis is more pronounced using diabetic fibrinogen and is related to glycaemic control

Fibrinolysis during clot formation In a purified system, and using pooled fibrinogen from diabetic and control individuals, the prolongation of lysis time after addition of $\mathrm{C} 3$ was greater in fibrin clots prepared from diabetic compared with control fibrinogen (increase of $244 \pm 64$ and $92 \pm 23 \mathrm{~s}$ respectively; $p<0.05$; Fig. $3 \mathrm{a}$ and $\mathrm{b}$ ).

Using a random pool of fibrinogen from seven samples before and after improving glycaemic control $\left(\mathrm{HbA}_{1 \mathrm{c}} 9.9 \pm\right.$ $0.5 \%$ and $8.0 \pm 0.3 \%$ [ $85 \pm 5$ and $64 \pm 3 \mathrm{mmol} / \mathrm{mol}]$ respectively; $p<0.01$ ), C3-induced prolongation of clot lysis time was longer at baseline (266 $\pm 108 \mathrm{~s})$ than following improvement in glycaemia $(211 \pm 94 \mathrm{~s})$, a difference that demonstrated a trend but failed to reach statistical significance ( $p=0.067, n=5$ experiments done in duplicate).
Fig. 3 Complement C3 prolongs clot lysis in a purified system. a Effects of $\mathrm{C} 3$ and bovine serum albumin (BSA) on fibrinolysis during clot formation using pooled fibrinogen purified from diabetes/control (DM/Co) samples ( $n=9$ each). Experiments were done in duplicate on six separate occasions. Grey dotted line, Co $\mathrm{BSA}$; grey solid line, Co C3; black dotted line, DM BSA; black solid line, DM C3. b C3induced prolongation of lysis time during clot formation using diabetes and control fibrinogen, compared with BSA as a control protein. c Real-time fibrinolysis of fully mature clots using pooled fibrinogen from $\mathrm{DM} / \mathrm{Co}$ in the presence of $\mathrm{C} 3$ and employing dual staining techniques. d C3-induced prolongation of lysis time of fully mature clots, using diabetes and control fibrinogen. e The effects of pooled diabetic (DM) and healthy control (Cont) $\mathrm{C} 3$ on prolongation of lysis time during clot formation using fibrinogen $(\mathrm{Fg})$ from healthy controls and diabetic patients. ${ }^{*} p<0.05$
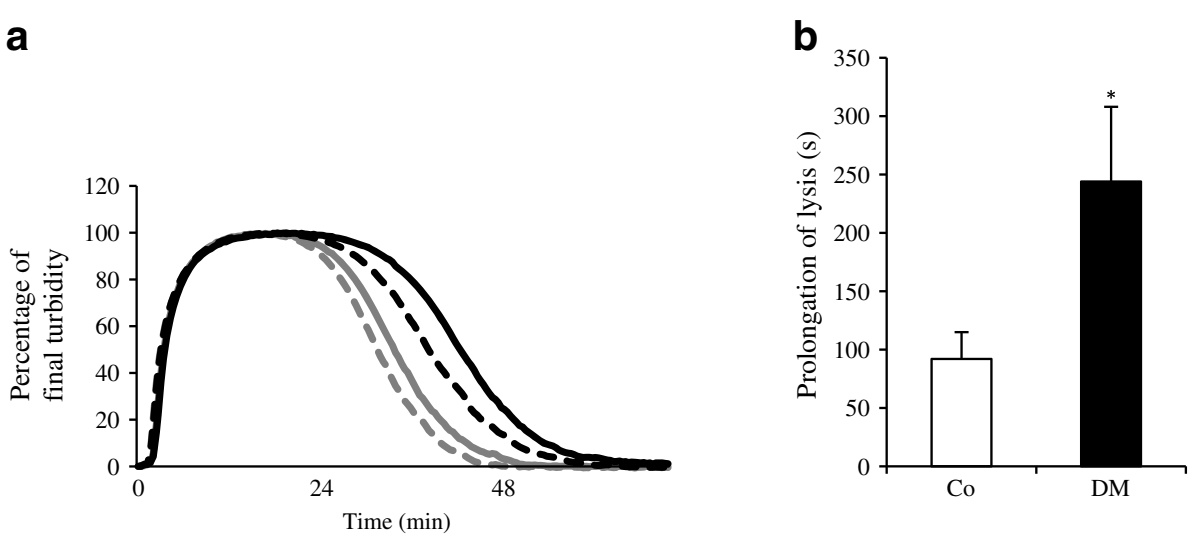

C
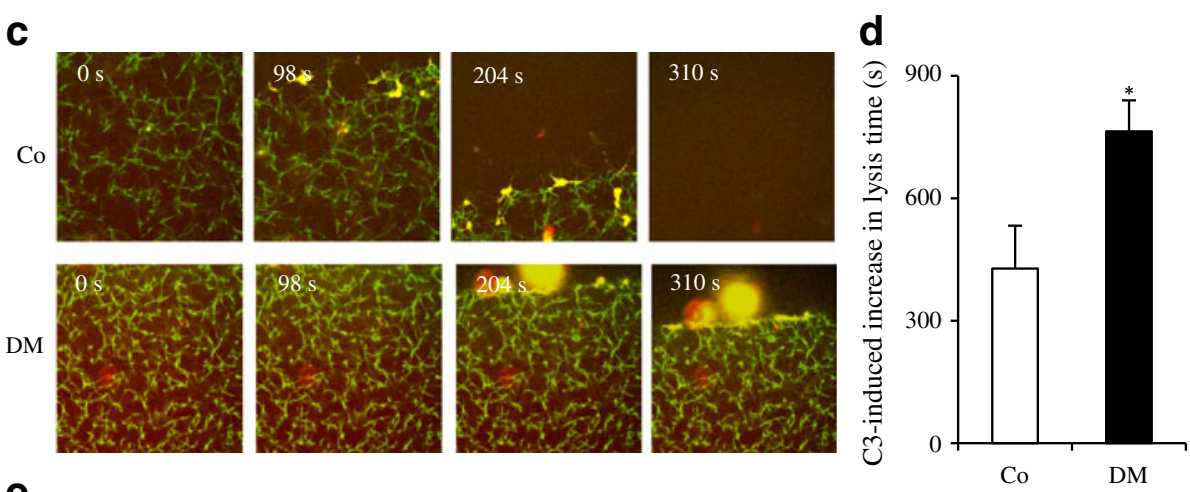

e
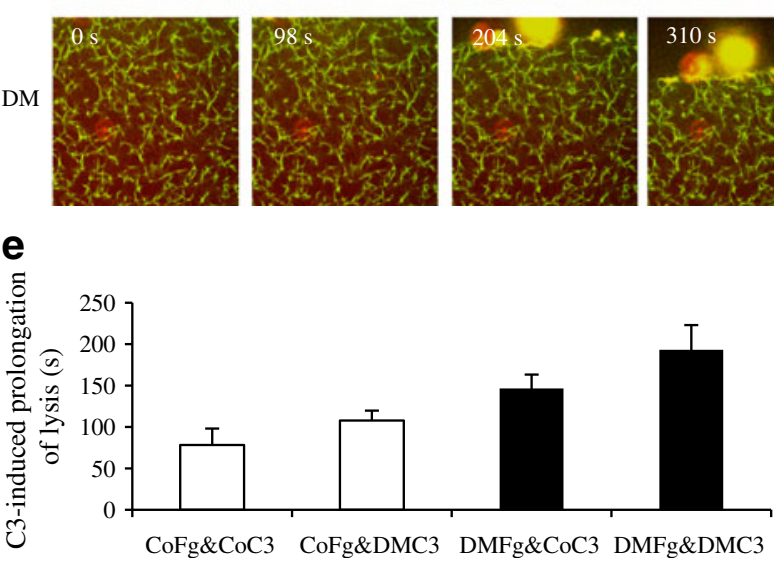
Fibrinolysis of fully formed clots This was investigated using confocal microscopy techniques. Similarly to the turbidity assay, real-time fibrinolysis demonstrated a more pronounced effect of $\mathrm{C} 3$ on lysis time in clots made from diabetic compared with control fibrinogen, resulting in fibrinolysis prolongation of $764 \pm 76$ and $428 \pm 105 \mathrm{~s}$ respectively; $p<0.05$ (Fig. $3 c$ and d).

\section{C3 from diabetic patients also prolongs clot lysis time}

We undertook preliminary work to investigate whether diabetic $\mathrm{C} 3$ has a similar effect on the fibrinolytic process compared with control protein. Employing the turbidimetric assay, pooled $\mathrm{C} 3$ purified from healthy controls $(n=6)$ and diabetic patients $\left(n=6, \mathrm{HbA}_{1 \mathrm{c}} 10.2 \pm 0.3 \%\right.$ [ $88 \pm 3 \mathrm{mmol} /$ mol]) prolonged lysis time in clots made from healthy control fibrinogen by $76 \pm 20$ and $108 \pm 12$ s respectively, whereas in clots made from diabetes fibrinogen, lysis prolongation was $147 \pm 17$ and $193 \pm 30$ s respectively. Although C3 prolongation of clot lysis by diabetic C3 appeared longer than control protein, the difference failed to reach statistical significance using control fibrinogen $(p>$ $0.1)$, but a trend was observed using diabetes fibrinogen $(p=$ 0.077). Experiments were repeated on four different occasions and results are summarised in Fig. 3e.

$\mathrm{C} 3$ is associated with the formation of thinner fibrin fibres

Turbidity curves demonstrated lower maximum absorbance in the presence of $\mathrm{C} 3$, suggesting thinner fibre formation (Fig. 4a). Scanning electron microscopy showed an average fibre diameter of clots made from control fibrinogen in the absence and presence of $\mathrm{C} 3$ of $81.3 \pm 3.0$ and $62.8 \pm 1.2 \mathrm{~nm}$ respectively $(p<0.01)$, whereas clots made from diabetes fibrinogen had a fibre diameter $61.4 \pm 1.3$ and $54.6 \pm 1.4 \mathrm{~nm}$ in the absence and presence of $\mathrm{C} 3$ respectively $(p<0.01$; Fig. $4 \mathrm{~b}-\mathrm{e})$. A significant difference was also detected comparing fibre thickness of clots made from control and diabetes fibrinogen in both the absence and presence of $\mathrm{C} 3(p<0.01)$.

Binding of $\mathrm{C} 3$ to diabetes and control fibrin(ogen) is similar

Concentration-dependent, high-affinity binding of $\mathrm{C} 3$ to immobilised control and diabetes fibrin and fibrinogen was observed. The interaction had a stoichiometry of $1: 1$, with no significant differences demonstrated $\left(K_{\mathrm{D}} \mathrm{C} 3\right.$ binding to control fibrin $0.39 \pm 0.05 \mathrm{nmol} / \mathrm{l}$, control fibrinogen $0.33 \pm$ $0.27 \mathrm{nmol} / 1$, diabetic fibrin $0.4 \pm 0.06 \mathrm{nmol} / 1$, diabetic fibrinogen $0.23 \pm 0.03 \mathrm{nmol} / \mathrm{l}$; Fig. $5 \mathrm{a}-\mathrm{d}$ ). Binding analysis performed in the reverse orientation showed again no statistically significant difference between control and diabetic fibrinogen (data not shown).
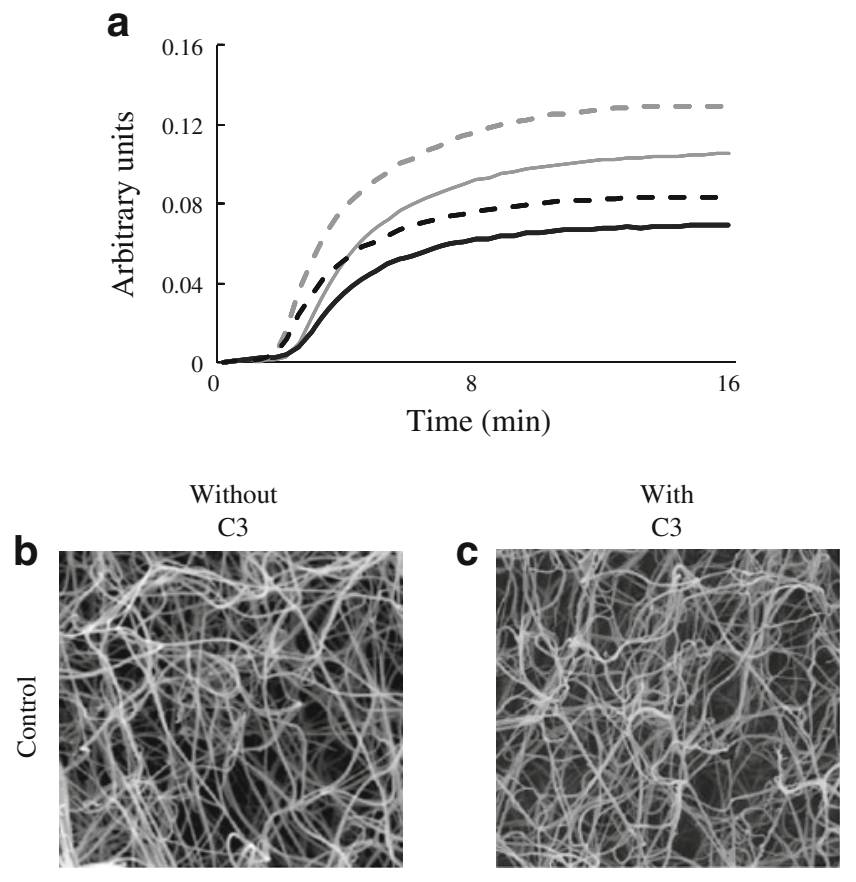

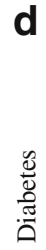
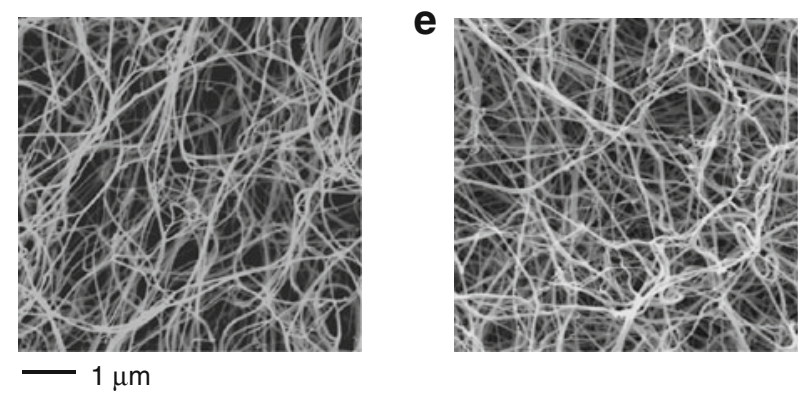

Fig. 4 The effects of complement $\mathrm{C} 3$ on fibrin network structure. a Turbidity curves of clots made from pooled purified diabetes and control fibrinogen in the presence of $\mathrm{C} 3$ or BSA as a control protein. Solid grey line, Co C3; dotted grey line, Co BSA; solid black line, DM C3; dotted black line, DM BSA. b-e Scanning electron micrographs (EM) of clots prepared from pooled control fibrinogen in the absence and presence of $\mathrm{C} 3$ (b and $\mathbf{c}$ respectively) or pooled diabetes fibrinogen in the absence and presence of $\mathrm{C} 3$ (d and e respectively)

\section{C3 does not affect plasmin generation}

Plasmin generation in the presence of control and diabetes fibrin without $\mathrm{C} 3$ was $0.176 \pm 0.009$ and $0.170 \pm$ $0.006 \mathrm{mg} \mathrm{l}^{-1} \mathrm{~min}^{-1}$ respectively. The addition of $\mathrm{C} 3$ to the reaction had no effect on plasmin generation in the presence of control or diabetes fibrin $(0.171 \pm 0.006$ and $0.169 \pm$ $0.01 \mathrm{mg} \mathrm{l}^{-1} \mathrm{~min}^{-1}$ respectively; $p>0.1$ for both).

\section{Discussion}

This work demonstrates impaired fibrinolysis in children with diabetes, and, given the absence of micro- and 
Fig. 5 Binding interaction between $\mathrm{C} 3$ and fibrin and fibrinogen. Binding of $\mathrm{C} 3$ to immobilised control fibrin and fibrinogen (a and $\mathbf{b}$ respectively), as well as diabetic fibrin and fibrinogen (c and $\mathbf{d}$ respectively). For each, a serial dilution of $0.019-10 \mathrm{nmol} / 1$, purified C3 was injected in an ascending order over the fibrin(ogen) surfaces. a $K_{\mathrm{D}}=0.39 \pm 0.05 \mathrm{nmol} / \mathrm{l}$; b $K_{\mathrm{D}}=0.33 \pm 0.27 \mathrm{nmol} / \mathrm{l}$; c $K_{\mathrm{D}}=0.4 \pm 0.06 \mathrm{nmol} / 1$ d $K_{\mathrm{D}}=0.23 \pm 0.03 \mathrm{nmol} / 1$ a

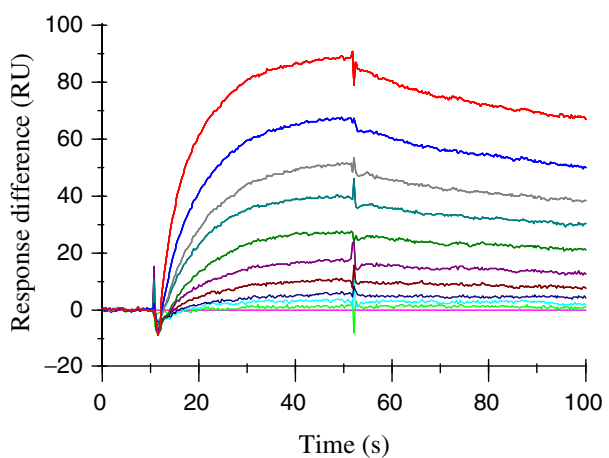

C

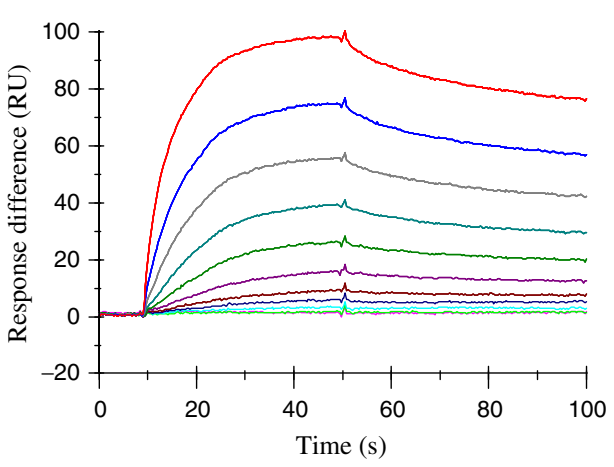

b

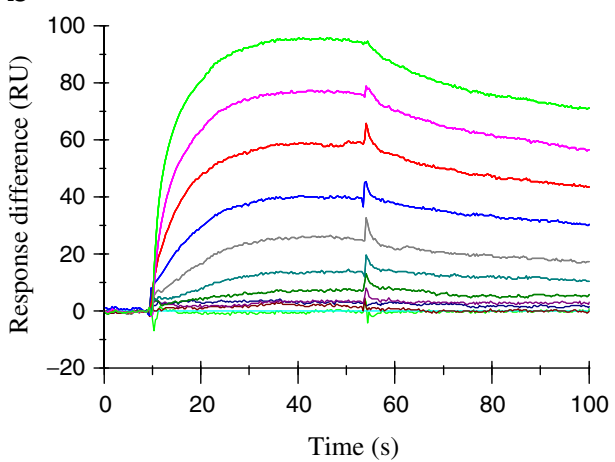

d

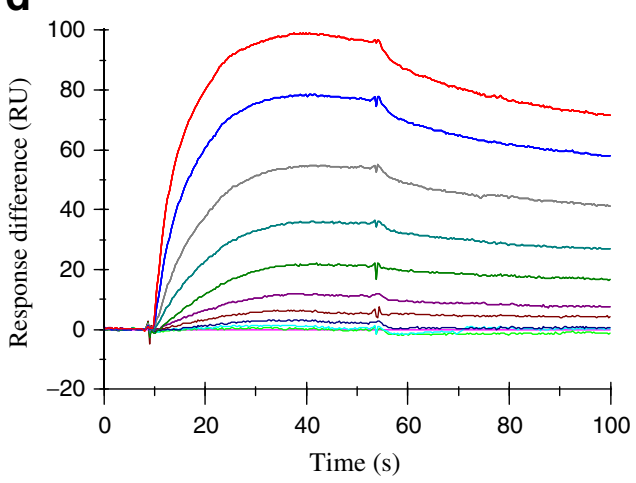

macrovascular complications in this young cohort, this observation may be attributed, at least in part, to elevated blood glucose levels. The concept is supported by shortening of lysis time after relatively modest improvement in medium-term glucose control in young adults with type 1 diabetes. C3 plasma levels were higher in children with diabetes compared with healthy controls and a reduction in C3 plasma levels was seen after improving glycaemia. Although CRP levels decreased, this failed to show statistical significance. This is consistent with previous work showing that improved glycaemic control in diabetes is associated with reduction in inflammatory markers [27]. Studies on C3 levels in autoimmune diabetes showed contradictory results ranging from elevated C3 [28, 29], to normal or reduced levels [30]. In the current study, samples were collected from individuals who had the condition for longer than 5 years, minimising the risk of an active autoimmune process interfering with C3 levels. The thrombotic environment in diabetic children was further evident by higher plasma fibrinogen compared with healthy controls, although protein levels were not affected by lowering blood glucose levels. These findings indicate that the prothrombotic/inflammatory milieu associated with diabetes and cardiovascular risk develops at an early stage in this metabolic disorder, and can be partly influenced by improving glycaemic control. Interestingly, the inflammatory component is mainly represented by $\mathrm{C} 3$, rather than
CRP, in the population studied. It should be noted that higher fibrinogen levels in diabetic children may have contributed to longer clot lysis, although modulation of lysis time and the lack of change in plasma fibrinogen after improving glycaemia, in the young adults, argues against this being the sole contributor of compromised clot lysis in the children group. The mechanisms for these observations may be related to amelioration of glucotoxicity-induced inflammation. It should be noted that optimising glycaemia is associated with alteration in lipid profile and reduced oxidative stress, which may influence the fibrinolytic process [31-33]. Also, extensive clinical input can reduce the risk of hypoglycaemia, which may contribute to ameliorating the thrombotic milieu [34, 35]. Therefore, glycaemia may affect the thrombotic/inflammatory environment through both direct and indirect mechanisms.

PAI-1, a key modulator of fibrinolysis [36], plasma levels correlated with clot lysis time. However, improving glycaemia had no effects on PAI-1 levels, indicating that the observed changes in clot lysis are PAI-1-independent. The temporal relationship between the fall in C3 plasma levels and enhanced fibrinolysis on improving glycaemic control indicates an interaction between $\mathrm{C} 3$ and the fibrinolytic system, a concept further supported by $\mathrm{C} 3$ induced prolongation of clot lysis, in turbidity and confocal experiments. Prolongation of clot lysis by C3 was more marked in clots made from diabetic compared with control 
fibrinogen, suggesting an enhanced effect in diabetes. A plausible mechanism for this observation may be related to increased incorporation of $\mathrm{C} 3$ into diabetic fibrin clots, supported by our ELISA and confocal microscopy data. Furthermore, our data suggest that $\mathrm{C} 3$ modulation of fibrinolysis is affected by glucose levels, as improving glycaemic control marginally attenuated the effects of $\mathrm{C} 3$ on clot lysis. Fibrin network formation exposes cryptic sites that become amenable to tPA and plasminogen binding [37], and C3 incorporation into the clot may affect these sites, thereby compromising fibrinolysis. The higher incorporation of $\mathrm{C} 3$ into diabetes clots may increase the 'mechanical resistance' of the clot to lysis. Alternatively, it may affect the availability of plasmin to interact with fibrin fibres, as $\mathrm{C} 3$ is a substrate for this fibrinolytic factor [38]. Binding of $\mathrm{C} 3$ to fibrin(ogen) from diabetic and control individuals was similar and, therefore, the increased C3 incorporation into diabetes clots is not related to higher affinity binding to fibrin(ogen) but probably due to a more complex interaction between $\mathrm{C} 3$ and clot formation, which requires further investigations. However, we should be cautious in our interpretation, as SPR has its limitations; protein immobilisation may create 'unnatural' binding sites making results interpretation problematic. Also, the low concentration of fibrinogen used in these experiments may lead to the formation of monomeric fibrin, which fails to fully represent the mature fibrin network [39]. The effect of $\mathrm{C} 3$ on plasminogen activation was similar in the presence of diabetic and control fibrinogen, thereby ruling this out as a potential mechanism for the observed difference.

The incorporation of $\mathrm{C} 3$ into the clot was associated with the formation of thinner fibres, which may be due to an effect of C3 on lateral aggregation. Changes in clot structure can influence fibrinolysis [4], but as these alterations occurred both in clots made from both diabetic and control fibrinogen, they are unlikely to explain the observed differences in lysis.

Diabetic and control C3 showed similar effects on lysis of clots made from control fibrinogen, although diabetic $\mathrm{C} 3$ had a possible additional effect on fibrinolysis of clots made from diabetes fibrinogen. Future work is needed using individual samples to investigate whether post-translational modifications in $\mathrm{C} 3$, related to glycaemic control, type of diabetes or therapy used, further modulate the magnitude of $\mathrm{C} 3$-induced prolongation of fibrinolysis.

In conclusion, this is the first study to demonstrate impaired fibrin clot lysis in children with diabetes and to directly implicate complement $\mathrm{C} 3$ as a new molecule that modulates the fibrinolytic process in this condition. A shorter lysis time and a reduction in $\mathrm{C} 3$ plasma levels were observed with improved glycaemic control. Ex vivo/in vitro work showed C3 incorporation into fibrin clots, directly compromising clot lysis, an effect that is enhanced in diabetes and can be ameliorated by improving glycaemia. More specifically, our data support an interaction between post-translation modifications (e.g. glycation) of fibrinogen and $\mathrm{C} 3$, being responsible for altered clot lysis properties in diabetes.

Therefore, $\mathrm{C} 3$ provides an important link between inflammation and thrombosis in diabetes, and represents a novel PAI-1 independent pathway for impaired fibrinolysis in this condition. Further studies are needed to clarify the precise mechanisms that underpin our findings, which may offer new therapeutic targets to reduce thrombotic events in this highrisk population.

Acknowledgements $\mathrm{K}$. Hess is supported by grants from the German ADUMED foundation and the Deutsche Forschungsgemeinschaft (DFG-HE 5666/1-1). S.H. Alzahrani is supported by King Fahad Medical City, Diabetes Center. V. Schroeder is supported by fellowships from the Swiss National Foundation and the Novartis Jubilee Foundation. We would like to express our gratitude to the NIHR and BHF for their generous funding. We are grateful to V. Ponnambalam (Faculty of Biological Sciences, University of Leeds, UK) for helpful discussions. We would also like to acknowledge the technical assistance of F. Phoenix (The LIGHT Laboratories, University of Leeds, UK).

Contribution statement $\mathrm{KH}$ designed and conducted experiments, interpreted data, wrote the manuscript. SA analysed and interpreted data, revised the manuscript. MM collected plasma samples, designed the study and revised the article. VS conducted experiments, interpreted data and revised the manuscript. AMC contributed to the study design and revised the article. GH conducted experiments, analysed data and helped with drafting the article. TK analysed and interpreted data and revised the article. MWJS contributed to design and data interpretation and revised the article. JFP contributed to design and data interpretation and revised the article. KAS conducted experiments, interpreted data and revised the article. PJG contributed to the conception and design of the study and revised the article. RAA designed and conducted experiments, interpreted data and wrote the manuscript. All authors approved the final version of the manuscript.

Duality of interest The authors declare that there is no duality of interest associated with this manuscript.

\section{References}

1. Berry C, Tardif JC, Bourassa MG (2007) Coronary heart disease in patients with diabetes: part I: recent advances in prevention and noninvasive management. J Am Coll Cardiol 49:631-642

2. Fatah K, Silveira A, Tornvall P, Karpe F, Blomback M, Hamsten A (1996) Proneness to formation of tight and rigid fibrin gel structures in men with myocardial infarction at a young age. Thromb Haemost 76:535-540

3. Undas A, Podolec P, Zawilska K et al (2009) Altered fibrin clot structure/function in patients with cryptogenic ischemic stroke. Stroke 40:1499-1501

4. Collet JP, Park D, Lesty C et al (2000) Influence of fibrin network conformation and fibrin fiber diameter on fibrinolysis speed: 
dynamic and structural approaches by confocal microscopy. Arterioscler Thromb Vasc Biol 20:1354-1361

5. Collet JP, Allali Y, Lesty C et al (2006) Altered fibrin architecture is associated with hypofibrinolysis and premature coronary atherothrombosis. Arterioscler Thromb Vasc Biol 26:2567-2573

6. Ajjan R, Lim BC, Standeven KF et al (2008) Common variation in the $\mathrm{C}$-terminal region of the fibrinogen betachain: effects on fibrin structure, fibrinolysis and clot rigidity. Blood 111:643-650

7. Jörneskog G, Egberg N, Fagrell B et al (1996) Altered properties of the fibrin gel structure in patients with IDDM. Diabetologia 39:1519-1523

8. Targher G, Chonchol M, Zoppini G, Franchini M (2011) Hemostatic disorders in type 1 diabetes mellitus. Semin Thromb Hemost 37:58-65

9. Jörneskog G, Hansson LO, Wallen NH, Yngen M, Blombäck M (2003) Increased plasma fibrin gel porosity in patients with type I diabetes during continuous subcutaneous insulin infusion. J Thromb Haemost 1:1195-1201

10. Pieters M, Covic N, du Loots $\mathrm{T}$ et al (2006) The effect of glycaemic control on fibrin network structure of type 2 diabetic subjects. Thromb Haemost 96:623-629

11. Ridker PM, Silvertown JD (2008) Inflammation, C-reactive protein, and atherothrombosis. J Periodontol 79:1544-1551

12. Ajjan R, Grant PJ, Futers TS et al (2005) Complement C3 and Creactive protein levels in patients with stable coronary artery disease. Thromb Haemost 94:1048-1053

13. Amara U, Flierl MA, Rittirsch D et al (2010) Molecular intercommunication between the complement and coagulation systems. J Immunol 185:5628-5636

14. Tedesco F, Fischetti F, Pausa M, Dobrina A, Sim RB, Daha MR (1999) Complement-endothelial cell interactions: pathophysiological implications. Mol Immunol 36:261-268

15. Shats-Tseytlina EA, Nair CH, Dhall DP (1994) Complement activation: a new participant in the modulation of fibrin gel characteristics and the progression of atherosclerosis? Blood Coagul Fibrinolysis 5:529-535

16. Distelmaier K, Adlbrecht C, Jakowitsch J et al (2009) Local complement activation triggers neutrophil recruitment to the site of thrombus formation in acute myocardial infarction. Thromb Haemost 102:564-572

17. Schroeder V, Carter AM, Dunne J, Mansfield MW, Grant PJ (2010) Proinflammatory and hypofibrinolytic phenotype in healthy first-degree relatives of patients with type 2 diabetes. J Thromb Haemost 8:2080-2082

18. Muscari A, Massarelli G, Bastagli L et al (2000) Relationship of serum $\mathrm{C} 3$ to fasting insulin, risk factors and previous ischaemic events in middle-aged men. Eur Heart J 21:1081-1090

19. Engström G, Hedblad B, Janzon L, Lindgärde F (2007) Complement $\mathrm{C} 3$ and $\mathrm{C} 4$ in plasma and incidence of myocardial infarction and stroke: a population-based cohort study. Eur J Cardiovasc Prev Rehabil 14:392-397

20. Carter AM, Prasad UK, Grant PJ (2009) Complement C3 and Creactive protein in male survivors of myocardial infarction. Atherosclerosis 203:538-543

21. Clauss A (1957) Rapid physiological coagulation method in determination of fibrinogen. Acta Haematol 17:237-246
22. Carter AM, Cymbalista CM, Spector TD, Grant PJ, EuroCLOT Investigators (2007) Heritability of clot formation, morphology, and lysis: the EuroCLOT study. Arterioscler Thromb Vasc Biol 27:2783-2789

23. Van den Berg CW, van Dijk H, Capel PJ (1989) Rapid isolation and characterization of native mouse complement components $\mathrm{C} 3$ and C5. J Immunol Methods 122:73-78

24. Deutsch DG, Mertz ET (1970) Plasminogen: purification from human plasma by affinity chromatography. Science 170:1095-1096

25. Smith KA, Adamson PJ, Pease RJ et al (2011) Interactions between factor XIII and the $\{$ alpha $\}$ C region of fibrinogen. Blood 117:3460-3468

26. Bobbink IW, Tekelenburg WL, Sixma JJ, de Boer HC, Banga JD, de Groot PG (1997) Glycated proteins modulate tissueplasminogen activator-catalyzed plasminogen activation. Biochem Biophys Res Commun 240:595-601

27. Heliövaara MK, Teppo AM, Karonen SL, Tuominen JA, Ebeling $P$ (2006) Improved glycaemia in type 1 diabetes results in decreased levels of soluble adhesion molecules with no change in serum adiponectin or most acute phase proteins. Exp Clin Endocrinol Diabetes 114:295-300

28. Morimoto Y, Taniguchi H, Yamashiro Y, Ejiri K, Baba S, Arimoto Y (1988) Complements in diabetes mellitus: activation of complement system evidenced by $\mathrm{C} 3 \mathrm{~d}$ elevation in IDDM. Diabetes Res Clin Pract 5:309-312

29. Sundsmo JS, Papin RA, Wood L et al (1985) Complement activation in type 1 human diabetes. Clin Immunol Immunopathol $35: 211-225$

30. Charlesworth JA, Timmermans V, Golding J et al (1987) The complement system in type 1 (insulin-dependent) diabetes. Diabetologia 30:372-379

31. Cannon CP (2008) Mixed dyslipidemia, metabolic syndrome, diabetes mellitus, and cardiovascular disease: clinical implications. Am J Cardiol 102:5L-9L

32. Giacco F, Brownlee M (2010) Oxidative stress and diabetic complications. Circ Res 107:1058-1070

33. Henschen-Edman AH (2001) Fibrinogen non-inherited heterogeneity and its relationship to function in health and disease. Ann N Y Acad Sci 936:580-593

34. Gogitidze Joy N, Hedrington MS, Briscoe VJ, Tate DB, Ertl AC, Davis SN (2010) Effects of acute hypoglycemia on inflammatory and pro-atherothrombotic biomarkers in individuals with type 1 diabetes and healthy individuals. Diabetes Care 33:1529-1535

35. Ajjan RA, Robinson EJ, Alzahrani SH, Grant PJ, Heller SR (2010) The effect of hypoglycemia on fibrin clot structure and fibrinolysis in individuals with type 1 diabetes. Diabetes 59 (Suppl 1):402-PP (Abstract)

36. Grant PJ (2007) Diabetes mellitus as a prothrombotic condition. J Intern Med 262:157-172

37. Medved L, Nieuwenhuizen W (2003) Molecular mechanisms of initiation of fibrinolysis by fibrin. Thromb Haemost 89:409-419

38. Seya T, Nagasawa S, Matsukura M, Hasegawa H, Atkinson JP (1985) Generation of C3d, g and C3d by urokinase-treated plasma in association with fibrinolysis. Complement 2:165-174

39. Tsurupa G, Yakovlev S, McKee P, Medved L (2010) Noncovalent interaction of alpha(2)-antiplasmin with fibrin(ogen): localization of alpha(2)-antiplasmin-binding sites. Biochemistry 49:7643-7651 